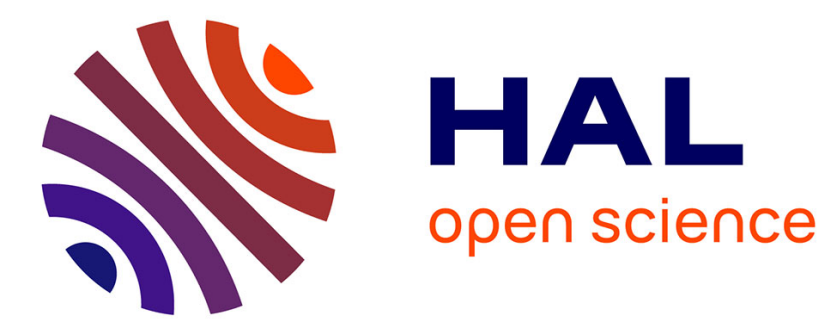

\title{
Bearing Rigidity Theory in SE(3)
}

Giulia Michieletto, Angelo Cenedese, Antonio Franchi

\section{To cite this version:}

Giulia Michieletto, Angelo Cenedese, Antonio Franchi. Bearing Rigidity Theory in SE(3). 55th IEEE Conference on Decision and Control, Dec 2016, Las Vegas, United States. hal-01371084

\section{HAL Id: hal-01371084 https://hal.science/hal-01371084}

Submitted on 23 Sep 2016

HAL is a multi-disciplinary open access archive for the deposit and dissemination of scientific research documents, whether they are published or not. The documents may come from teaching and research institutions in France or abroad, or from public or private research centers.
L'archive ouverte pluridisciplinaire HAL, est destinée au dépôt et à la diffusion de documents scientifiques de niveau recherche, publiés ou non, émanant des établissements d'enseignement et de recherche français ou étrangers, des laboratoires publics ou privés. 


\title{
Bearing Rigidity Theory in SE(3)
}

\author{
Giulia Michieletto, Angelo Cenedese, and Antonio Franchi
}

\begin{abstract}
Rigidity theory has recently emerged as an efficient tool in the control field of coordinated multi-agent systems, such as multi-robot formations and UAVs swarms, that are characterized by sensing, communication and movement capabilities. This work aims at describing the rigidity properties for frameworks embedded in the three-dimensional Special Euclidean space $S E(3)$ wherein each agent has 6DoF. In such scenario, it is assumed that the devices are able to gather bearing measurements w.r.t. their neighbors, expressing them into their own body frame. The goal is then to identify the framework transformations that allow to preserve such measurements maintaining it rigid. Rigidity properties are mathematically formalized in this work which differs from the previous ones as it faces the extension in three-dimensional space dealing with the 3D rotations manifold. In particular, the attention is focused on the infinitesimal $S E(3)$-rigidity for which a necessary and sufficient condition is provided.
\end{abstract}

\section{INTRODUCTION}

The term rigidity usually concerns a combinatorial theory for characterizing the stiffness of structures formed by rigid bodies connected by flexible linkages or hinges [1]. Specifically, the goal is studying whether one can deform the shape of such type of structure preserving the relative poses among the agents in terms of distances and/or directions.

For this reason, rigidity theory is involved in the properties evaluation of several systems deriving from different fields of research, e.g., mechanical and building structures, biological and artificial compounds, industrial materials, and recently, it has taken an outstanding position in the interactive multiagent context [2]-[4]. Indeed, rigidity framework fits for applications connected to the motion control of mobile robots and to the sensors cooperation for localization, exploration, mapping and tracking of a target. More generally, it turns out to be an important architectural property of many multiagent systems where a common inertial reference frame is unavailable but the agents involved are characterized by sensing, communication and movement capabilities.

According to the available sensing measurements, rigidity properties for a framework deals with inter-agent distance and/or direction maintenance. When agents are able to gather only range information, distance constraints can be imposed to preserve distance rigidity properties. On the other hand, parallel/bearing rigidity properties are determined by direction contraints defined upon angle/bearing measurements.

Main notions about distance rigidity are illustrated in [5][7]: these works explain how distance constraints for a framework can be summarized into a unique matrix whose rank

G. Michieletto and A. Cenedese are with the Department of Information Engineering, University of Padova, Padova, Italy, giulia.michieletto@unipd.it, angelo.cenedese@unipd.it.

A. Franchi is with LAAS-CNRS, Université de Toulouse, CNRS, Toulouse, France, afranchi@laas.fr determines the infinitesimal rigidity properties of the system, providing a necessary and sufficient condition. In such a context, a framework is generally represented by means of the bar-and-joint model where agents are represented as points joined by bars whose fixed lengths enforce the interagent distance constraints. Infinitesimal rigidity properties concern with distance-preserving motions of the framework which thus consist of only global roto-translations of the entire system as a rigid body.

As far as bearing rigidity in $\mathbb{R}^{2}$ (or parallel rigidity) is concerned, it is determined by the definition of normal constraints over the directions of neighboring agents, namely the edges of the sensing/communication graph associated to the framework, as explained in [8]-[13]. These constraints entail the preservation of the angles formed between pairs of agents and the lines joining them, i.e., the inter-agent bearings. As a consequence, allowed collective infinitesimal motions consist of only global translations and uniform scaling of the whole set of positions in the framework.

The same infinitesimal motions characterize the infinitesimally bearing rigid frameworks in $\mathbb{R}^{d}$ with $d>2$, where the bearing measurement between agents $i$ and $j$ coincides with the angle between the $x$-axis of the local coordinate system of node $i$ and the line segment joining node $i$ with node $j$, as stated in [13], [14]. In literature, some distinctions are usually made between the $2 \mathrm{D}$ case and the higher dimensional ones, although in [13] a unified dissertation for bearing rigidity is presented. Several theoretical results are also provided in [13], however the main contribution is the necessary and sufficient condition to guarantee that a given framework is infinitesimally bearing rigid in $\mathbb{R}^{d}, d \geq 2$. This is related to the rank and eigenvalues of a certain matrix which summarizes the involved constraints.

In [11], [12], bearing rigidity theory has been studied for systems embedded in $S E(2)$, i.e., frameworks where the underlying graph is directed and bearings are expressed in the local frame of each agent of the framework modeled as a $S E(2)$-point having its own position and attitude in the plane. Because of the nature of the $S E(2)$ manifold, bearing-preserving infinitesimal motions for a given framework are composed of motions in $\mathbb{R}^{2}$ with motions in $\mathbb{S}^{1}$ (1-dimensional manifold on the unit circle). Hence, these result to be rigid-body translations and uniform scaling of the whole set of positions but also coordinated rotations, namely the synchronized rotations of all the agents about their own body axis at the same angular speed, coupled with a rigid-body rotation of the whole framework in $\mathbb{R}^{2}$.

The current state of the art motivates the study of bearing rigidity for formations in $S E(3)$, which is the main contribution of this work. As for $S E(2)$ case, the principal features 
of such theory concern the bearing measurements that are referred to the local frame of each agent $(S E(3)$-point having its own position and attitude in the $3 \mathrm{D}$ space) corroborating the use of a directed graph to represent agents interactions. Nevertheless, the extension from the two-dimensional Special Euclidean space to the three-dimensional one is not trivial. The main challenge deals with the attitude representation of the agents: while in $S E(2)$ a single angle is sufficient to determine the orientation of a robot on the plane, in $3 \mathrm{D}$ space three degrees of freedom have to be managed involving different and more complex type of manifolds. In this sense, even though the infinitesimal motions are still translations, uniform scaling and coordinated rotations, they have been redefined in the new higher-dimensional context. In addition, it has been proven that they span the null-space of the so called $S E$ (3)-rigidity matrix and, similarly to [11], that a framework is infinitesimally rigid in $S E(3)$ if and only if the size of the null-space of this matrix is equal to seven.

The paper is organized as follows. The notation used is depicted in Sec. II and a brief review of the bearing rigidity theory in $\mathbb{R}^{3}$ is provided in Sec. III. Sec. IV is devoted to the development of rigidity theory for $S E(3)$, clearly illustrating the properties of allowed infinitesimal motions, while some examples are given in Sec. V. Finally, concluding remarks and future research directions are discussed in Sec. VI.

\section{PRELIMINARIES AND NotATION}

In this work, $S E(3)=\mathbb{R}^{3} \times S O(3)$ indicates the threedimensional Special Euclidean group describing the rigidbody transformations in 3D space. Specifically, $S O(3)$ refers to the $3 \mathrm{D}$ rotation group, while the 2 -dimensional manifold on the unit sphere in $\mathbb{R}^{3}$ is denoted as $\mathbb{S}^{2}$. Note that $S O(3)$ is not isomorphic to $\mathbb{S}^{2}$, while in $2 \mathrm{D}$ space it holds $S O(2) \simeq \mathbb{S}^{1}$.

The standard Euclidean norm of a vector $\mathbf{v} \in \mathbb{R}^{3}$ is indicated as $\|\mathbf{v}\|$ so that $\|\mathbf{v}\|^{2}=\mathbf{v}^{\top} \mathbf{v}$. The cross product between the vectors $\mathbf{v}, \mathbf{u} \in \mathbb{R}^{3}$ is instead computed as $\mathbf{v} \times \mathbf{u}=\mathbf{S}(\mathbf{v}) \cdot \mathbf{u}$, where $\mathbf{S}(\cdot)$ is the map that associates any vector in $\mathbb{R}^{3}$ to its corresponding skew-symmetric matrix belonging to the Special Orthogonal Lie algebra, $\mathfrak{s o}(3)$.

A directed graph $\mathscr{G}=(\mathscr{V}, \mathscr{E})$ is specified by a vertex set $\mathscr{V}$ and an edge set $\mathscr{E} \subseteq \mathscr{V} \times \mathscr{V}$, such that $|\mathscr{V}|=n$ and $|\mathscr{E}|=$ $m$. The graph with all the possible directed edges, i.e., the complete directed graph with $m=n(n-1)$, is denoted as $K_{n}$. The relationship between the sets $\mathscr{V}$ and $\mathscr{E}$ is described by the incidence matrix $\mathbf{E} \in \mathbb{R}^{n \times m}$, namely the $\{0, \pm 1\}$-matrix such that

$$
[\mathbf{E}]_{i k}=\left\{\begin{array}{lll}
1 & \text { if } \quad e_{k}=\left(v_{i}, v_{j}\right) \in \mathscr{E} & \text { (outgoing edge) } \\
-1 & \text { if } \quad e_{k}=\left(v_{j}, v_{i}\right) \in \mathscr{E} & \text { (ingoing edge) } \\
0 & \text { otherwise } &
\end{array}\right.
$$

Similarly, the $\{0,1\}$-matrix $\mathbf{E}_{\text {out }} \in \mathbb{R}^{n \times m}$ is designed so that

$$
\left[\mathbf{E}_{\text {out }}\right]_{i k}= \begin{cases}1 & \text { if } e_{k}=\left(v_{i}, v_{j}\right) \in \mathscr{E} \quad \text { (outgoing edge) } \\ 0 & \text { otherwise }\end{cases}
$$

The notation $\overline{\mathbf{E}}=\mathbf{E} \otimes \mathbf{I}_{3} \in \mathbb{R}^{3 n \times 3 m}$ and $\overline{\mathbf{E}}_{\text {out }}=\mathbf{E}_{\text {out }} \otimes \mathbf{I}_{3} \in$ $\mathbb{R}^{3 n \times 3 m}$ is also introduced.
Finally, given a generic matrix $\mathbf{A} \in \mathbb{R}^{p \times q}$, its null-space is indicated as $\mathscr{N}(\mathbf{A})$ and its (column) rank as $r k(\mathbf{A})$, while $\operatorname{diag}\left(\left\{\mathbf{A}_{i}\right\}\right) \in \mathbb{R}^{r p \times r q}$ refers to a block diagonal concatenation of all the matrices $\left\{\mathbf{A}_{i} \in \mathbb{R}^{p \times q}, i=1, \ldots, r\right\}$.

\section{BEARING RIGIDITY THEORY IN $\mathbb{R}^{3}$}

Some fundamental concepts of bearing rigidity are here briefly reviewed, focusing on frameworks embedded in $\mathbb{R}^{3}$. A more exhaustive dissertation is reported in [13].

A framework in $\mathbb{R}^{3}$, denoted as $(\mathscr{G}, \mathbf{p})$, is a pair consisting of an undirected graph $\mathscr{G}=(\mathscr{V}, \mathscr{E})$ and a configuration $\mathbf{p}=$ $\left[\mathbf{p}_{1}^{\top} \ldots \mathbf{p}_{n}^{\top}\right]^{\top} \in \mathbb{R}^{3 n}$ such that each vertex $v_{i} \in \mathscr{V}$ in the graph is associated to the point $\mathbf{p}_{i} \in \mathbb{R}^{3}$. In other words, it is a set of $n$ agents able to interact according to the the edge set $\mathscr{E}$, each of them with a specific position $\mathbf{p}_{i}$ into the three-dimensional space. As a consequence, for each pair $\left(v_{i}, v_{j}\right) \in \mathscr{E}$ the unit vector $\overline{\mathbf{p}}_{i j}=\mathbf{p}_{i j} /\left\|\mathbf{p}_{i j}\right\|$, where $\mathbf{p}_{i j}=\mathbf{p}_{j}-\mathbf{p}_{i}$, represents the relative bearing between $v_{i}$ and $v_{j}$ expressed w.r.t. some common reference frame.

Bearing rigidity theory in $\mathbb{R}^{3}$ aims at determining when a framework can be uniquely determined up to a translation and a uniform scaling (bearing-preserving transformations) by the set of inter-neighbor bearings.

In order to tackle this issue, it is advantageous to define the orthogonal projector operator $\mathbf{P}: \mathbb{R}^{3} \rightarrow \mathbb{R}^{3 \times 3}$, which geometrically projects any nonzero vector $\mathbf{v} \in \mathbb{R}^{3}$ onto its orthogonal complement. Explicitly it holds

$$
\mathbf{P}(\mathbf{v})=\mathbf{I}_{3}-\frac{\mathbf{v} \mathbf{v}^{\top}}{\|\mathbf{v}\|^{2}} .
$$

The resulting orthogonal projection matrix $\mathbf{P}(\mathbf{v})$ is symmetric, idempotent and positive semi-definite, and its nullspace coincides with the space generated by vector $\mathbf{v}$, i.e., $\mathscr{N}(\mathbf{P}(\mathbf{v}))=\operatorname{span}\{\mathbf{v}\}$.

The orthogonal projection operator provides a convenient way to describe parallel vectors in $\mathbb{R}^{3}$ where the bearing rigidity notion relies on the the parallelism conservation for the line segment between any pair of nodes of a framework. Indeed, two frameworks $(\mathscr{G}, \mathbf{p})$ and $\left(\mathscr{G}, \mathbf{p}^{\prime}\right)$ are said to be bearing equivalent if $\mathbf{P}\left(\mathbf{p}_{i}-\mathbf{p}_{j}\right) \cdot\left(\mathbf{p}_{i}^{\prime}-\mathbf{p}_{j}^{\prime}\right)=\mathbf{0}$ for all $\left(v_{i}, v_{j}\right) \in \mathscr{E}$ and bearing congruent if $\mathbf{P}\left(\mathbf{p}_{i}-\mathbf{p}_{j}\right) \cdot\left(\mathbf{p}_{i}^{\prime}-\mathbf{p}_{j}^{\prime}\right)=\mathbf{0}$ for all $v_{i}, v_{j} \in \mathscr{V}$. Hence, two bearing congruent frameworks are always related by an appropriate finite sequence of rigidbody translations and scaling.

Definition 1 (Bearing Rigidity [13, Def. 3]). A framework $(\mathscr{G}, \mathbf{p})$ is bearing rigid if there exists an $\varepsilon>0$ such that every framework $\left(\mathscr{G}, \mathbf{p}^{\prime}\right)$ which is bearing equivalent to $(\mathscr{G}, \mathbf{p})$ and satisfies $\left\|\mathbf{p}_{i}-\mathbf{p}_{i}^{\prime}\right\|<\varepsilon$ for all $v_{i} \in \mathscr{V}$, is bearing congruent to $(\mathscr{G}, \mathbf{p})$.

Definition 2 (Global Bearing Rigidity [13, Def. 4]). A framework $(\mathscr{G}, \mathbf{p})$ is globally bearing rigid if all bearing equivalent frameworks to $(\mathscr{G}, \mathbf{p})$ are also bearing congruent to $(\mathscr{G}, \mathbf{p})$.

All the bearings in a given framework can be summarized through the bearing rigidity function $\mathbf{b}_{\|, \mathscr{G}}: \mathbb{R}^{3 n} \rightarrow \mathbb{R}^{3 m}$ that maps the configuration $\mathbf{p}$ into the stacked bearings vector, 


\begin{tabular}{|c|c|c|c|c|}
\hline Node domains & Measurement domains & Measurement properties & Nomenclature & References \\
\hline $\mathbf{p}_{i} \in \mathbb{R}^{d}$ & $\begin{array}{c}\mathbf{d}_{i j}=\left\|\mathbf{p}_{i}-\mathbf{p}_{j}\right\| \in \mathbb{R}^{d} \\
\mathbf{b}_{i j}=\frac{\mathbf{p}_{j}-\mathbf{p}_{i}}{\left\|\mathbf{p}_{i}-\mathbf{p}_{j}\right\|} \in \mathbb{S}^{d-1}\end{array}$ & $\begin{array}{c}\mathbf{d}_{j i}=\mathbf{d}_{i j} \text { (undirected underlying graph) } \\
\mathbf{b}_{j i}=-\mathbf{b}_{i j} \text { (undirected underlying graph) }\end{array}$ & $\begin{array}{c}\text { (distance) rigidity } \\
\text { parallel/bearing rigidity }\end{array}$ & [13], [8], [9], [10] \\
\hline $\boldsymbol{\chi}_{i}=\left(\mathbf{p}_{i}, \theta_{i}\right) \in S E(2)$ & $\mathbf{b}_{i j}=\mathbf{R}\left(\theta_{i}\right)^{\top} \frac{\mathbf{p}_{j}-\mathbf{p}_{i}}{\left\|\mathbf{p}_{i}-\mathbf{p}_{j}\right\|} \in \mathbb{S}^{1}$ & $\mathbf{b}_{i j}, \mathbf{b}_{i j}$ independent (directed underlying graph) & $S E(2)$-rigidity & [11], [12] \\
\hline $\boldsymbol{\chi}_{i}=\left(\mathbf{p}_{i}, \mathbf{R}_{i}\right) \in S E(3)$ & $\mathbf{b}_{i j}=\mathbf{R}_{i}^{\top} \frac{\mathbf{p}_{j}-\mathbf{p}_{i}}{\left\|\mathbf{p}_{i}-\mathbf{p}_{j}\right\|} \in \mathbb{S}^{2}$ & $\mathbf{b}_{i j}, \mathbf{b}_{i j}$ independent (directed underlying graph) & $S E(3)$-rigidity & \\
\hline
\end{tabular}

TABLE I: Summary of the state of the art about rigidity properties: different theories have been formulated according to nodes domain, measurements domain and properties.

i.e., $\mathbf{b}_{\|, \mathscr{G}}(\mathbf{p})=\left[\begin{array}{lll}\overline{\mathbf{p}}_{1}^{\top} & \ldots \overline{\mathbf{p}}_{m}^{\top}\end{array}\right]^{\top}$ where $\overline{\mathbf{p}}_{k}$ refers to the measurement on the $k$-th edge. The Jacobian of the bearing function $\mathbf{B}_{\|, \mathscr{G}}(\mathbf{p}) \in \mathbb{R}^{3 m \times 3 n}$, which is referred as bearing rigidity matrix, represents a key feature to study the rigidity properties of a framework in $\mathbb{R}^{3}$. Indeed, considering a variation $\boldsymbol{\delta}_{p}$ of the configuration $\mathbf{p}$, if $\mathbf{B}_{\|, \mathscr{G}}(\mathbf{p}) \boldsymbol{\delta}_{p}=\mathbf{0}$, then $\boldsymbol{\delta}_{p}$ is called an infinitesimal bearing motion of $(\mathscr{G}, \mathbf{p})$. Moreover, if an infinitesimal motion corresponds to a bearing-preserving transformation, namely a translation or a scaling of the entire framework, it is referred as trivial.

Definition 3 (Infinitesimal Bearing Rigidity [13, Def. 5]). A framework $(\mathscr{G}, \mathbf{p})$ is infinitesimally bearing rigid if every possible infinitesimal motion is trivial.

The infinitesimal bearing rigidity properties of a framework are linked to the null-space and the rank of the corresponding bearing rigidity matrix.

Theorem 1 (Condition for Infinitesimal Bearing Rigidity [13, Thm. 4]). For a framework $(\mathscr{G}, \mathbf{p})$ in $\mathbb{R}^{3}$, the following statements are equivalent:

(a) $(\mathscr{G}, \mathbf{p})$ is infinitesimally bearing rigid;

(b) $r k\left(\mathbf{B}_{\|, \mathscr{G}}(\mathbf{p})\right)=3 n-4$;

(c) $\mathscr{N}\left(\mathbf{B}_{\|, \mathscr{G}}(\mathbf{p})\right)=\operatorname{span}\left\{\mathbf{1} \otimes \mathbf{I}_{3}, \mathbf{p}\right\}$.

Moreover, it can be proven that the infinitesimal bearing rigidity is a stronger property than both bearing and global bearing rigidity which are equivalent.

Theorem 2 (Rigidity Properties). For a framework $(\mathscr{G}, \mathbf{p})$ in $\mathbb{R}^{3}$, the following implications are valid:

- bearing global rigidity $\Leftrightarrow$ bearing rigidity [13, Thm. 3];

- bearing infinitesimally rigidity $\Rightarrow$ bearing global rigidity [13, Thm. 5];

- bearing infinitesimally rigidity $\Rightarrow$ bearing rigidity.

Some main features of the bearing rigidity in $\mathbb{R}^{3}$ can be derived from the Tab. I that provides an overview on the taxonomy used in the rigidity literature according to the domain of nodes and measurements.

\section{Rigidity ThEORY IN SE(3)}

The concepts introduced in previous section are required to extend the rigidity theory for frameworks that are embedded in the three-dimensional Special Euclidean group $S E(3)$. Here, each agent is characterized by a position and an attitude in the 3D space and is assumed to interact with its neighors according to a certain directed graph.

Definition 4 (SE(3) framework). An $S E$ (3) framework is a triple $(\mathscr{G}, \mathbf{p}, \mathbf{a})$, where $\mathscr{G}=(\mathscr{V}, \mathscr{E})$ is a directed graph, $\mathbf{p}: \mathscr{V} \rightarrow \mathbb{R}^{3}$ is a function mapping each node to a point in $\mathbb{R}^{3}$ (position) and $\mathbf{a}: \mathscr{V} \rightarrow S O(3)$ is a function associating each node with an element of $S O(3)$ (attitude).

In literature various well-known formalisms exist to represent the attitude of an agent able to move in 3D space, e.g., Euler angles, quaterions, axis-angle representation. In this work, the agents orientation is expressed through the rotation matrices which belong to the Special Orthogonal group $S O(3)$. This group includes all the $3 \times 3$ orthogonal matrices having unit determinant. Therefore, in the following, the attitude of each agent $i$ has to be interpreted as a rotation matrix $\mathbf{R}_{i} \in \mathbb{R}^{3 \times 3}$ such that $\mathbf{R}_{i} \mathbf{R}_{i}^{T}=\mathbf{I}_{3}$ and $\operatorname{det}\left(\mathbf{R}_{i}\right)=+1$.

Hereafter, for notation convenience, the position and attitude of the node $v_{i} \in \mathscr{V}$ is denoted by $\left(\mathbf{p}\left(v_{i}\right), \mathbf{a}\left(v_{i}\right)\right)=$ $\left(\mathbf{p}_{i}, \mathbf{R}_{i}\right)=\left(\boldsymbol{\chi}_{p}(i), \boldsymbol{\chi}_{a}(i)\right)=\boldsymbol{\chi}(i) \in S E(3)$, whereas $\mathbf{p}(\mathscr{V})=$ $\chi_{p}(\mathscr{V}) \in \mathbb{R}^{3 n}$ and $\mathbf{a}(\mathscr{V})=\chi_{a}(\mathscr{V}) \in S O(3)^{n}$ indicate the position and attitude components of the complete framework configuration, respectively.

Similarly to the $S E(2)$ case (see [11], [12] for details), the extension to $S E(3)$ explicitly handles frameworks where the underlying graph is directed and bearings are expressed in the local body frame of each agent ( $S E(3)$ point). These assumptions are justified by real multi-agent scenarios where a robot can gather the relative bearings between itself and other robots through sensors attached to its body frame such as, e.g., robots flying in 3D with onboard cameras.

In this venue, the agent $i$ accesses the bearing of the agent $j$ if and only if the directed edge $\left(v_{i}, v_{j}\right)$ belongs to the graph $\mathscr{G}$. Furthermore, the relative bearing $\mathbf{b}_{i j} \in \mathbb{S}^{2}$ is measured from the body coordinate system of the $i$-th agent, but it can be expressed in terms of the relative positions and attitudes of the two points w.r.t. the world frame. It holds

$$
\mathbf{b}_{i j}=\mathbf{R}_{i}^{\top} \frac{\mathbf{p}_{i}-\mathbf{p}_{j}}{\left\|\mathbf{p}_{i}-\mathbf{p}_{j}\right\|}=\mathbf{R}_{i}^{\top} \overline{\mathbf{p}}_{i j},
$$

where the matrix $\mathbf{R}_{i}^{\top}$ is the rotation matrix describing the orientation of the world frame w.r.t. the body frame of agent $i$, and $\overline{\mathbf{p}}_{i j}$ is a shorthand notation for the normalized relative position vector from $i$ to $j$.

Bearing rigidity theory in $S E(3)$ seeks to evaluate the rigidity properties of a given framework $(\mathscr{G}, \mathbf{p}, \mathbf{a})$ which rest on its bearing measurements preservation. In other words, the main goal is to identify the allowed motions that do not modify the whole systems in term of inter-agent bearings.

Definition 5 (Equivalence and Congruence in $S E(3)$ ). Two frameworks $(\mathscr{G}, \mathbf{p}, \mathbf{a})$ and $\left(\mathscr{G}, \mathbf{p}^{\prime}, \mathbf{a}^{\prime}\right)$ are equivalent if

$$
\mathbf{R}_{i}^{\top} \frac{\mathbf{p}_{i}-\mathbf{p}_{j}}{\left\|\mathbf{p}_{i}-\mathbf{p}_{j}\right\|}=\mathbf{R}_{i}^{\prime \top} \frac{\mathbf{p}_{i}^{\prime}-\mathbf{p}_{j}^{\prime}}{\left\|\mathbf{p}_{i}^{\prime}-\mathbf{p}_{j}^{\prime}\right\|} \quad \forall\left(v_{i}, v_{j}\right) \in \mathscr{E} .
$$


Two frameworks $(\mathscr{G}, \mathbf{p}, \mathbf{a})$ and $(\mathscr{G}, \mathbf{s}, \mathbf{0})$ are congruent if

$$
\mathbf{R}_{i}^{\top} \frac{\mathbf{p}_{i}-\mathbf{p}_{j}}{\left\|\mathbf{p}_{i}-\mathbf{p}_{j}\right\|}=\mathbf{R}_{i}^{\prime \top} \frac{\mathbf{p}_{i}^{\prime}-\mathbf{p}_{j}^{\prime}}{\left\|\mathbf{p}_{i}^{\prime}-\mathbf{p}_{j}^{\prime}\right\|} \quad \forall v_{i}, v_{j} \in \mathscr{V} .
$$

Definition 6 (Global Rigidity in $S E(3)$ ). A framework $(\mathscr{G}, \mathbf{p}, \mathbf{a})$ is globally rigid in $S E(3)$ if every framework which is equivalent to $(\mathscr{G}, \mathbf{p}, \mathbf{a})$ is also congruent to $(\mathscr{G}, \mathbf{p}, \mathbf{a})$.

The information about bearing measurements can be handled defining the $S E(3)$-rigidity function associated to the framework, namely the function $\mathbf{b} \mathscr{G}: S E(3)^{n} \rightarrow \mathbb{S}^{2^{m}}$ such that

$$
\mathbf{b}_{\mathscr{G}}(\boldsymbol{\chi}(\mathscr{V}))=\left[\mathbf{b}_{1}^{\top} \ldots \mathbf{b}_{m}^{\top}\right]^{\top},
$$

where $\mathbf{b}_{k}$ denotes the measurement on the $k$-th directed edge in the graph $\mathscr{G}$. It can be proven that the $S E(3)$-rigidity function can be rewritten in the more compact form

$$
\mathbf{b}_{\mathscr{G}}(\boldsymbol{\chi}(\mathscr{V}))=-\operatorname{diag}\left(\left\{\frac{\mathbf{R}_{i}^{\top}}{\left\|\mathbf{p}_{i}-\mathbf{p}_{j}\right\|}\right\}\right) \overline{\mathbf{E}}^{\top} \boldsymbol{\chi}_{p}(\mathscr{V}) .
$$

In such a scenario, a framework $(\mathscr{G}, \mathbf{p}, \mathbf{a})$ is $S E$ (3)-rigid if and only if for any $\chi^{\prime}(\mathscr{V}) \in S E(3)^{n}$ sufficiently close to $\chi(\mathscr{V})$ with the same bearing measurements, namely $\mathbf{b}_{\mathscr{G}}(\boldsymbol{\chi}(\mathscr{V}))=\mathbf{b}_{\mathscr{G}}\left(\boldsymbol{\chi}^{\prime}(\mathscr{V})\right)$, there exists a (local) bearingpreserving transformation taking $\boldsymbol{\chi}(\mathscr{V})$ to $\boldsymbol{\chi}^{\prime}(\mathscr{V})$.

Definition 7 (Rigidity in $S E(3))$. A framework $(\mathscr{G}, \mathbf{p}, \mathbf{a})$ is rigid in $S E(3)$ if there exists a neighborhood $S \subset S E(3)^{n}$ of $\boldsymbol{\chi}(\mathscr{V})$ such that

$$
\mathbf{b}_{K_{n}}^{-1}\left(\mathbf{b}_{K_{n}}(\boldsymbol{\chi}(\mathscr{V}))\right) \cap S=\mathbf{b}_{\mathscr{G}}^{-1}\left(\mathbf{b}_{\mathscr{G}}(\boldsymbol{\chi}(\mathscr{V}))\right) \cap S,
$$

where $\mathbf{b}_{K_{n}}^{-1}\left(\mathbf{b}_{K_{n}}(\boldsymbol{\chi}(\mathscr{V}))\right)$ denotes the pre-image of the point $\mathbf{b}_{K_{n}}(\boldsymbol{\chi}(\mathscr{V}))$ under the SE(3)-rigidity map.

The set $\mathbf{b}_{K_{n}}^{-1}\left(\mathbf{b}_{K_{n}}(\boldsymbol{\chi}(\mathscr{V}))\right) \subset S E(3)^{n}$ contains $\boldsymbol{\chi}(\mathscr{V})$ and all its possible transformations induced by the complete graph $K_{n}$, while the set $\mathbf{b}_{\mathscr{G}}^{-1}\left(\mathbf{b}_{\mathscr{G}}(\boldsymbol{\chi}(\mathscr{V}))\right) \subset S E(3)^{n}$ contains (at least) $\boldsymbol{\chi}(\mathscr{V})$ and all its possible transformations induced by the graph $\mathscr{G}$. Thus, $\mathbf{b}_{\mathscr{G}}^{-1}\left(\mathbf{b}_{\mathscr{G}}(\boldsymbol{\chi}(\mathscr{V}))\right)-\mathbf{b}_{K_{n}}^{-1}\left(\mathbf{b}_{K_{n}}(\boldsymbol{\chi}(\mathscr{V}))\right)$ is the set of all the possible transformations of $\boldsymbol{\chi}(\mathscr{V})$ constrained by $\mathscr{G}$ that are not admissible by $K_{n}$ and allows to define the property of roto-flexibility for a framework.

Definition 8 (Roto-flexibility in $S E(3)$ ). A framework $(\mathscr{G}, \mathbf{p}, \mathbf{a})$ is roto-flexible in $S E(3)$ if there exists an analytic path $\eta:[0,1] \rightarrow S E(3)^{n}$ such that $\eta(0)=\chi(\mathscr{V})$ and $\eta(t) \in$ $\mathbf{b}_{\mathscr{G}}^{-1}\left(\mathbf{b}_{\mathscr{G}}(\boldsymbol{\chi}(\mathscr{V}))\right)-\mathbf{b}_{K_{n}}^{-1}\left(\mathbf{b}_{K_{n}}(\boldsymbol{\chi}(\mathscr{V}))\right)$ for all $t \in(0,1]$.

Using the language of Def. 8 , a smooth motion along a path $\eta$ with $\eta(0)=\boldsymbol{\chi}(\mathscr{V})$ such that the initial rate of change of the rigidity function is zero (bearing measurements do not change), constitutes an infinitesimal motion of a $S E(3)$ framework. It can be proven that the set of infinitesimal motions is characterized by the null-space of the Jacobian of the $S E$ (3)-rigidity function, namely by the null-space of the $S E(3)$-rigidity matrix $\mathbf{B}_{\mathscr{G}}(\boldsymbol{\chi}(\mathscr{V}))=\nabla_{\chi} \mathbf{b}_{\mathscr{G}}(\boldsymbol{\chi}(\mathscr{V})) \in \mathbb{R}^{3 m \times 6 n}$ such that

$$
\begin{aligned}
& \frac{\partial \mathbf{b}_{i j}}{\partial \boldsymbol{\chi}(i)}=\left[\begin{array}{ll}
\frac{\partial \mathbf{b}_{i j}}{\partial \mathbf{p}_{i}} & \frac{\partial \mathbf{b}_{i j}}{\partial \mathbf{R}_{i}}
\end{array}\right]=\left[\begin{array}{ll}
-\frac{\mathbf{P}\left(\mathbf{b}_{i j}\right)}{\left\|\mathbf{p}_{i}-\mathbf{p}_{j}\right\|} \mathbf{R}_{i}^{\top} & S\left(\mathbf{b}_{i j}\right)
\end{array}\right], \\
& \frac{\partial \mathbf{b}_{i j}}{\partial \boldsymbol{\chi}(j)}=\left[\begin{array}{ll}
\frac{\partial \mathbf{b}_{i j}}{\partial \mathbf{p}_{j}} & \frac{\partial \mathbf{b}_{i j}}{\partial \mathbf{R}_{j}}
\end{array}\right]=\left[\begin{array}{ll}
\frac{\mathbf{P}\left(\mathbf{b}_{i j}\right)}{\left\|\mathbf{p}_{i}-\mathbf{p}_{j}\right\|} \mathbf{R}_{i}^{\top} & \mathbf{0}
\end{array}\right] .
\end{aligned}
$$

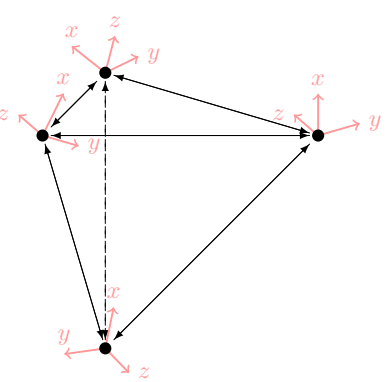

Fig. 1: Example of infinitesimally rigid $S E(3)$ framework, where $n=4$, the underlying graph $\mathscr{G}$ is complete $(m=6)$, each agent has its own body frame.

As a consequence, if a path $\eta$ is contained entirely in $\mathbf{b}_{K_{n}}^{-1}\left(\mathbf{b}_{K_{n}}(\boldsymbol{\chi}(\mathscr{V}))\right)$ for all $t \in[0,1]$, then it correspond to an infinitesimal motion. Hence, the infinitesimal motions are completely described by the tangent bundle $T_{p}$ of $\mathbf{b}_{K_{n}}^{-1}\left(\mathbf{b}_{K_{n}}(\boldsymbol{\chi}(\mathscr{V}))\right)$ and it holds $T_{p}=\mathscr{N}\left[\mathbf{B}_{K_{n}}(\boldsymbol{\chi}(\mathscr{V}))\right]$.

These premises lead to a formal definition for infinitesimal rigidity of frameworks in $S E(3)$.

Definition 9 (Infinitesimal Rigidity in $S E(3)$ ). A framework $(\mathscr{G}, \mathbf{p}, \mathbf{a})$ is infinitesimally rigid in $S E(3)$ if $\mathscr{N}\left[\mathbf{B}_{\mathscr{G}}(\boldsymbol{\chi}(\mathscr{V}))\right]=$ $\mathscr{N}\left[\mathbf{B}_{K_{n}}(\boldsymbol{\chi}(\mathscr{V}))\right]$. Otherwise it is infinitesimally roto-flexible in $\operatorname{SE}(3)$.

Def. 9 suggests that any $S E(3)$ framework whose underlying graph is complete, i.e., $\left(K_{n}, \mathbf{p}, \mathbf{a}\right)$ where $\mathbf{p}$ and $\mathbf{a}$ are suitable defined, is infinitesimally rigid (see Fig. 1); however it is not a necessary condition as explained in the following.

\section{A. Infinitesimal Motions Properties}

The main challenge regarding the extension of the rigidity theory to $S E(3)$ rests upon the identification of the bearingpreserving transformation in the $3 \mathrm{D}$ rotation manifold. Indeed, infinitesimal motions of a $S E(3)$ framework are the composition of motions in $\mathbb{R}^{3}$ with motions in $S O(3)$ for each point. Each infinitesimal motion $\boldsymbol{\delta}_{\chi} \in \mathscr{N}\left(\mathbf{B}_{\mathscr{G}}(\boldsymbol{\chi}(\mathscr{V}))\right)$ is therefore characterized by the velocity component $\boldsymbol{\delta}_{\chi_{p}} \in \mathbb{R}^{3 n}$ and the angular velocity component $\boldsymbol{\delta}_{\chi_{a}} \in \mathbb{R}^{3 n}$.

If all agents do not alter their attitude, i.e., $\boldsymbol{\delta}_{\chi_{a}}=0$, then the condition $\boldsymbol{\delta}_{\chi} \in \mathscr{N}\left(\mathbf{B}_{\mathscr{G}}(\boldsymbol{\chi}(\mathscr{V}))\right)$ reduces to the constraints for bearing rigidity in $\mathbb{R}^{3}$. Hence translations and uniform scaling of the framework result to be infinitesimal motions still in $S E(3)$ context. On the contrary, if the angular velocities of the agents are non-zero, then the only infinitesimal motions of the framework consists of the bearing-preserving motion termed $(3 D)$ coordinated rotation. It envisages a $3 \mathrm{D}$ synchronized rotation of all the agents in their frameworks jointly to an equal rotation of the whole framework as a rigidbody in $\mathbb{R}^{3}$ (the angular velocity of each agent is equal to the one of the center of mass of the system). Fig. 2 proposes an explanatory example of the just described infinitesimal motions.

To characterize these inifitesimal motions in $S E(3)$, it is suitable to introduce the permutated $S E(3)$-rigidity matrix $\widetilde{\mathbf{B}}_{\mathscr{G}}(\boldsymbol{\chi}(\mathscr{V}))$ that results from the permutation of the columns of $\mathbf{B}_{\mathscr{G}}(\boldsymbol{\chi}(\mathscr{V}))$ so as to be 


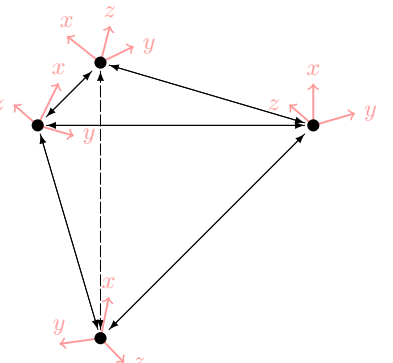

(a) $\operatorname{SE}(3)$ framework

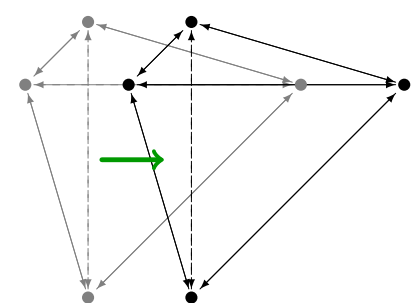

(b) Traslation

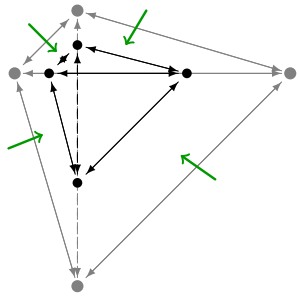

(c) Factor scaling

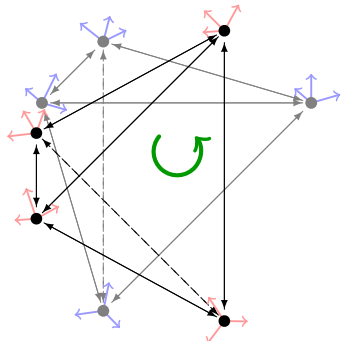

(d) Coordinated rotation

Fig. 2: Example of infinitesimal motions: the $S E$ (3) framework $\left(K_{4}, \mathbf{p}, \mathbf{a}\right)$ (Fig. 2a) is translated along the $x$ axis (Fig. 2b), scaled by a factor $\alpha=0.5$ (Fig. 2c), counterclockwise coordinated rotated of $\theta=\pi / 4 \mathrm{rad}$ (Fig. 2d).

$$
\begin{aligned}
& \widetilde{\mathbf{B}}_{\mathscr{G}}(\boldsymbol{\chi}(\mathscr{V}))=\left[\begin{array}{ll}
\nabla_{\boldsymbol{\chi}_{p}} \mathbf{b}_{\mathscr{G}}(\boldsymbol{\chi}(\mathscr{V})) & \nabla_{\boldsymbol{\chi}_{a}} \mathbf{b}_{\mathscr{G}}(\boldsymbol{\chi}(\mathscr{V}))
\end{array}\right] \\
& =\left[\operatorname{diag}\left(\left\{-\frac{\mathbf{P}\left(\mathbf{b}_{i j}\right)}{\left\|\mathbf{p}_{i}-\mathbf{p}_{j}\right\|} \mathbf{R}_{i}^{\top}\right\}\right) \cdot \overline{\mathbf{E}}^{\top} \quad \operatorname{diag}\left(\left\{\mathbf{S}\left(\mathbf{b}_{i j}\right)\right\}\right) \cdot \overline{\mathbf{E}}_{\text {out }}^{\top}\right] .
\end{aligned}
$$

Proposition 1. Every infinitesimal motion $\boldsymbol{\delta}_{\chi} \in \mathscr{N}\left[\mathbf{B}_{\mathscr{G}}(\chi(\mathscr{V}))\right]$ satisfies

$\operatorname{diag}\left(\left\{-\frac{\mathbf{P}\left(\mathbf{b}_{i j}\right)}{\left\|\mathbf{p}_{i}-\mathbf{p}_{j}\right\|} \mathbf{R}_{i}^{\top}\right\}\right) \overline{\mathbf{E}}^{\top} \boldsymbol{\delta}_{\chi_{p}}+\operatorname{diag}\left(\left\{\mathbf{S}\left(\mathbf{b}_{i j}\right)\right\}\right) \overline{\mathbf{E}}_{\text {out }}^{\top} \boldsymbol{\delta}_{\chi_{a}}=\mathbf{0}$.

Proof. The null-space $\mathscr{N}\left(\widetilde{\mathbf{B}}_{\mathscr{G}}\right)$ derive from the permutation of the vectors in the null-space of $\mathbf{B} g$. Therefore, the thesis is proved evaluating the permuted $S E(3)$-rigidity matrix.

Corollary 1. Every infinitesimal motion $\boldsymbol{\delta}_{\chi} \in \mathscr{N}\left[\mathbf{B}_{\mathscr{G}}(\chi(\mathscr{V}))\right]$ satisfies the condition

$$
\mathbf{B}_{\|, \mathscr{G}}\left(\boldsymbol{\chi}_{p}\right) \boldsymbol{\delta}_{\chi_{p}}=-\mathbf{F}\left(\boldsymbol{\chi}_{p}\right) \boldsymbol{\delta}_{\chi_{a}},
$$

where

$$
\begin{aligned}
\text { - } & \mathbf{B}_{\|, \mathscr{G}}\left(\boldsymbol{\chi}_{p}\right)=\operatorname{diag}\left(\left\{\frac{\mathbf{P}\left(\overline{\mathbf{p}}_{i j}\right)}{\left\|\mathbf{p}_{i}-\mathbf{p}_{j}\right\|}\right\}\right) \overline{\mathbf{E}}^{\top} \text { is the bearing rigidity } \\
& \text { matrix in } \mathbb{R}^{3} \text { (see Sec. III), } \\
\text { - } & \mathbf{F}\left(\boldsymbol{\chi}_{p}(\mathscr{V})\right)=-\operatorname{diag}\left(\left\{\mathbf{R}_{i} \mathbf{S}\left(\mathbf{b}_{i j}\right)\right\}\right) \overline{\mathbf{E}}_{\text {out }}^{\top} .
\end{aligned}
$$

Proof. The result is obtained directly by Prop. 1 through substitution.

Proposition 2. For the complete directed graph $K_{n}$, the coordinated rotation subspace $\mathscr{R}_{\circlearrowleft}\left(K_{n}\right)$ is not trivial and $\operatorname{dim} \mathscr{R}_{\circlearrowleft}\left(K_{n}\right)=3$.

Proof. Consider a rigid-body rotation $\boldsymbol{\delta}_{\chi}$ of the framework $\boldsymbol{\chi}$ with angular velocity $\boldsymbol{\omega}^{\prime}$. In a (3D) coordinated rotation, for each $i$-th agent the linear velocity $\dot{\mathbf{p}}_{i}$ results from the cross product between its position $\mathbf{p}_{i}$ and $\boldsymbol{\omega}^{\prime}$, while its angular velocity $\boldsymbol{\omega}_{i}$ is the expression of $\boldsymbol{\omega}^{\prime}$ in its body frame. Formally

$$
\begin{aligned}
& {\left[\boldsymbol{\delta}_{\chi_{p}}\right]_{i}=\dot{\mathbf{p}}_{i}=-\mathbf{S}\left(\mathbf{p}_{i}\right) \boldsymbol{\omega}^{\prime},} \\
& {\left[\boldsymbol{\delta}_{\chi_{a}}\right]_{i}=\boldsymbol{\omega}_{i}=\mathbf{R}_{i}^{\top} \boldsymbol{\omega}^{\prime} .}
\end{aligned}
$$

From Prop. 1, for the infinitesimal motions it must result

$$
\frac{\mathbf{P}\left(\mathbf{b}_{i j}\right)}{\left\|\mathbf{p}_{i}-\mathbf{p}_{j}\right\|} \mathbf{R}_{i}^{\top}\left(\dot{\mathbf{p}}_{i}-\dot{\mathbf{p}}_{j}\right)+\mathbf{S}\left(\mathbf{b}_{i j}\right) \boldsymbol{\omega}_{i}=\mathbf{0} \quad \forall v_{i}, v_{j} \in \mathscr{V}
$$

and, substituting (1)-(2), it is possible to verify that

$$
\begin{gathered}
\frac{\mathbf{P}\left(\mathbf{b}_{i j}\right)}{\left\|\mathbf{p}_{i}-\mathbf{p}_{j}\right\|} \mathbf{R}_{i}^{\top}\left(\mathbf{S}\left(\mathbf{p}_{i}\right) \boldsymbol{\omega}^{\prime}-\mathbf{S}\left(\mathbf{p}_{j}\right) \boldsymbol{\omega}^{\prime}\right)+\mathbf{S}\left(\mathbf{b}_{i j}\right) \mathbf{R}_{i}^{\top} \boldsymbol{\omega}^{\prime}= \\
=-\mathbf{P}\left(\mathbf{b}_{i j}\right) \mathbf{R}_{i}^{\top} \mathbf{S}\left(\overline{\mathbf{p}}_{i j}\right) \boldsymbol{\omega}^{\prime}+\mathbf{S}\left(\mathbf{R}_{i}^{\top} \overline{\mathbf{p}}_{i j}\right) \mathbf{R}_{i}^{\top} \boldsymbol{\omega}^{\prime}= \\
=-\mathbf{P}\left(\mathbf{b}_{i j}\right) \mathbf{S}\left(\mathbf{R}_{i}^{\top} \overline{\mathbf{p}}_{i j}\right) \mathbf{R}_{i}^{\top} \boldsymbol{\omega}^{\prime}+\mathbf{S}\left(\mathbf{R}_{i}^{\top} \overline{\mathbf{p}}_{i j}\right) \mathbf{R}_{i}^{\top} \boldsymbol{\omega}^{\prime}= \\
=\left(\mathbf{I}_{3}-\mathbf{P}\left(\mathbf{b}_{i j}\right)\right) \mathbf{S}\left(\mathbf{R}_{i}^{\top} \overline{\mathbf{p}}_{i j}\right) \mathbf{R}_{i}^{\top} \boldsymbol{\omega}^{\prime}=\mathbf{0} .
\end{gathered}
$$

Hence, the coordinated rotation subspace is not trivial as it contains all the vectors whose components satisfy (1) (2). Moreover, each arbitrary rigid-body rotation can be expressed as composition of rotations around the $x$-axis with angular velocity $\boldsymbol{\omega}^{\prime}=\left[\begin{array}{lll}1 & 0 & 0\end{array}\right]^{\top}$, the $y$-axis with angular velocity $\boldsymbol{\omega}^{\prime}=\left[\begin{array}{lll}0 & 1 & 0\end{array}\right]^{\top}$ and the $z$-axis with angular velocity $\boldsymbol{\omega}^{\prime}=\left[\begin{array}{lll}0 & 0 & 1\end{array}\right]^{\top}$. As a consequence, $\operatorname{dim} \mathscr{R}_{\circlearrowleft}\left(K_{n}\right)=3$.

Since Prop. 2 provides an analytic description for the vectors spanning the coordinated rotations subspace $\mathscr{R}_{\circlearrowleft}(\cdot)$, it is possible to exhaustively explicate the space of infinitesimal motions.

Corollary 2. For an infinitesimally rigid SE(3) framework,

$$
\mathscr{N}\left[\widetilde{\mathbf{B}}_{\mathscr{G}}(\boldsymbol{\chi}(\mathscr{V}))\right]=\operatorname{span}\left\{\left[\begin{array}{c}
\mathbb{1}_{n} \otimes \mathbf{I}_{3} \\
\mathbf{0}
\end{array}\right],\left[\begin{array}{c}
\boldsymbol{\chi}_{p} \\
\mathbf{0}
\end{array}\right], \mathscr{R}_{\circlearrowleft}(\mathscr{G})\right\} .
$$

Finally, the main result of this section relates the infinitesimal rigidity of an $S E(3)$ framework to the rank of the $S E(3)$-rigidity matrix.

Theorem 3. A SE(3) framework is infinitesimally rigid if and only if $r k[\mathbf{B} \mathscr{G}(\boldsymbol{\chi}(\mathscr{V}))]=6 n-7$.

Proof. It holds

$$
\begin{aligned}
r k\left[\mathbf{B}_{\mathscr{G}}(\boldsymbol{\chi}(\mathscr{V}))\right] & =r k\left[\widetilde{\mathbf{B}}_{\mathscr{G}}(\boldsymbol{\chi}(\mathscr{V}))\right] \\
& =\operatorname{dim}\left(\widetilde{\mathbf{B}}_{\mathscr{G}}(\boldsymbol{\chi}(\mathscr{V}))\right)-\mathscr{N}\left[\widetilde{\mathbf{B}}_{\mathscr{G}}(\boldsymbol{\chi}(\mathscr{V}))\right] \\
& =6 n-7,
\end{aligned}
$$

where the equivalence $\mathscr{N}\left[\widetilde{\mathbf{B}}_{\mathscr{G}}(\boldsymbol{\chi}(\mathscr{V}))\right]=7$ is justified by Cor. 2.

The same conclusion can be derived by considering the bearing rigidity matrix in $\mathbb{R}^{3}$, introduced in Sec. III.

Corollary 3. An $S E(3)$ framework is infinitesimally rigid in $S E(3)$ if and only if 


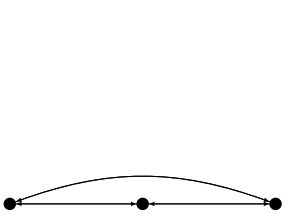

(a)

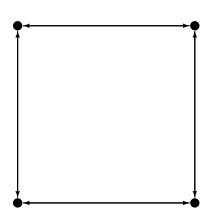

(b)

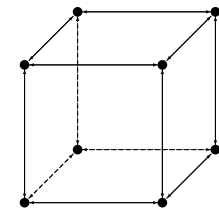

(c)
Fig. 3: Examples of non-infinitesimally $S E(3)$-rigid frameworks.

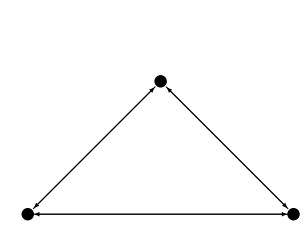

(a)

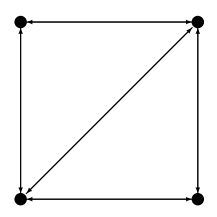

(b)

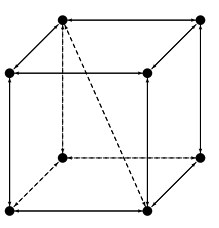

(c)
Fig. 4: Examples of infinitesimally $S E(3)$-rigid frameworks.

1) $r k\left[\mathbf{B}_{\| . \mathscr{G}}\left(\chi_{p}\right)\right]=3 n-4$,

2) $\operatorname{dim}_{\circlearrowleft}(\mathscr{G})=3$.

Proof. The statement follows directly from the Def. 9 of infinitesimal rigidity in $S E(3)$, Cor. 1 and Prop. 2.

Cor. 3 also allows to prove the necessary and sufficient condition for infinitesimal rigidity as follows.

Proof. of Theorem 3 Assume that $r k\left[\mathbf{B}_{\mathscr{G}}(\chi(\mathscr{V}))\right]=6 n-$ 7. From Cor. 1 and Prop. 2, it can be concluded that $r k\left[\mathbf{B}_{K_{n}}(\chi(\mathscr{V}))\right]=6 n-7$. By definition of infinitesimal rigidity in $S E(3)$, the framework $(\mathscr{G}, \mathbf{p}, \mathbf{a})$ is infinitesimally rigid. On the contrary, assuming that the framework $(\mathscr{G}, \mathbf{p}, \mathbf{a})$ is infinitesimally rigid in $S E(3)$, from Cor. 3 it follows that $r k\left[\mathbf{B}_{\|, \mathscr{G}}\left(\chi_{p}\right)\right]=3 n-4$ and $\operatorname{dim} \mathscr{R}_{\circlearrowleft}(\mathscr{G})=3$. Therefore, $r k[\mathbf{B} \mathscr{G}(\chi(\mathscr{V}))]=6 n-7$.

\section{NUMERICAL EXAMPLES}

In order to clarify the previous statements and definitions about infinitesimal rigidity in $S E(3)$, this section is devoted to some numerical examples. In detail, Fig. 3 shows examples of non-infinitesimally $S E$ (3)-rigid frameworks. On the other hand, Fig. 4 displays examples of infinitesimally $S E$ (3)-rigid frameworks, for which it can be verified $r k\left(\mathbf{B}_{\mathscr{G}}(\boldsymbol{\chi})\right)=6 n-7$. All the links depicted are bidirectional, meaning linked agents are able to reciprocally gather the bearing measurements.

It is worth to notice that when the agents are aligned the infinitesimal $S E(3)$-rigidity property is not ensured, while it could be attained by adding a single link in the other situations.

\section{CONCLUSions And Future Works}

This work introduces an extension of the rigidity theory to the case of frameworks embedded in $S E(3)$. The main contribution is the characterization of infinitesimal rigidity properties that are related to the rank of the $S E(3)$-rigidity matrix. Moreover, it has been showed that the null-space of that matrix describes the infinitesimal motions of an $S E(3)$ framework, including the rigid body translations and uniform scaling, in addition to (3D) coordinated rotations.

A considerable effort has been made in defining the concepts known in the rigidity literature for manifold dedicated to the representation of the rigid-bodies transformations in three-dimensional space. Nonetheless, this work aims to be the starting point for many future works concerning both the relative positions and orientation estimation and the control of 3D formation, e.g., UAVs swarms.

\section{REFERENCES}

[1] L. Asimow and B. Roth, "The rigidity of graphs, II," Journal of Mathematical Analysis and Applications, vol. 68, no. 1, pp. 171-190, 1979.

[2] R. Williams, A. Gasparri, A. Priolo, and G. Sukhatme, "Distributed combinatorial rigidity control in multi-agent networks," in Decision and Control (CDC), 2013 IEEE 52nd Annual Conference on, Dec 2013, pp. 6061-6066.

[3] N. Roy, P. Newman, and S. Srinivasa, Rigidity Maintenance Control for Multi-Robot Systems. MIT Press, 2013, pp. 504. [Online]. Available: http://ieeexplore.ieee.org/xpl/articleDetails.jsp? arnumber $=6577954$

[4] R. K. Williams, A. Gasparri, M. Soffietti, and G. S. Sukhatme, "Redundantly rigid topologies in decentralized multi-agent networks," in 2015 54th IEEE Conference on Decision and Control (CDC), Dec 2015, pp. 6101-6108.

[5] B. Jackson, "Notes on the rigidity of graphs," in Levico Conference Notes, vol. 4. Citeseer, 2007.

[6] D. Zelazo, A. Franchi, F. Allgöwer, H. H. Bülthoff, and P. Robuffo Giordano, "Rigidity maintenance control for multi-robot systems." in Robotics: Science and Systems. Sydney, Australia, 2012, pp. $473-480$.

[7] D. Zelazo, A. Franchi, H. H. Bülthoff, and P. Robuffo Giordano, "Decentralized rigidity maintenance control with range measurements for multi-robot systems," The Int. Journal of Robotics Research, vol. 34, no. 1, pp. 105-128, 2014.

[8] T. Eren, W. Whiteley, P. N. Belhumeur, B. Anderson et al., "Sensor and network topologies of formations with direction, bearing, and angle information between agents," in Decision and Control, 2003. Proceedings. 42nd IEEE Conference on, vol. 3. IEEE, 2003, pp. 3064-3069

[9] A. Franchi and P. Robuffo Giordano, "Decentralized control of parallel rigid formations with direction constraints and bearing measurements," in Decision and Control (CDC), 2012 IEEE 51st Annual Conference on. IEEE, 2012, pp. 5310-5317.

[10] T. Eren, "Using angle of arrival (bearing) information for localization in robot networks," Turk J Elec Engin, vol. 15, no. 2, 2007.

[11] D. Zelazo, A. Franchi, and P. Robuffo Giordano, "Rigidity theory in se (2) for unscaled relative position estimation using only bearing measurements," in Control Conference (ECC), 2014 European. IEEE, 2014, pp. 2703-2708.

[12] D. Zelazo, P. Robuffo Giordano, and A. Franchi, "Bearing-only formation control using an se(2) rigidity theory," in 54rd IEEE Conference on Decision and Control, Osaka, Japan, 12/2015 2015.

[13] S. Zhao and D. Zelazo, "Bearing rigidity and almost global bearingonly formation stabilization," IEEE Transactions on Automatic Control, vol. 61, no. 5, pp. 1255-1268, 2016

[14] - "Bearing-based distributed control and estimation of multi-agent systems," in Control Conference (ECC), 2015 European, 2015, pp. 2202-2207. 